\title{
Impairment of Arterial Compliance in Cushing's Syndrome
}

\author{
Pier Paolo Bassareo, ${ }^{1}$ Angela Maria Zedda ${ }^{2}$ and Giuseppe Mercuro ${ }^{3}$
}

1. Chief of the Section of Paediatric Cardiology, Department of Medical Sciences 'M Aresu', University of Cagliari, Cagliari, Italy; 2. Specialist Registrar in Cardiology, Department of Medical Sciences 'M Aresu', University of Cagliari, Cagliari, Italy; 3. Professor of Cardiology, Department of Medical Sciences 'M Aresu', University of Cagliari, Cagliari, Italy and Medical Director of the Cardiology and Angiology Unit, University Hospital of Cagliari, Italy

\begin{abstract}
Arterial stiffness may be useful for stratifying cardiovascular risk in individuals suffering from a number of pathologies, such as hypertension, diabetes, obesity, dyslipidaemia and coronary artery disease. Cushing's syndrome is underpinned by a complex metabolic syndrome, which is potentially implicated in the onset of blood vessel alterations and the increase in arterial wall stiffness. The aim of this paper was to perform a review about the most important studies conducted in order to evaluate the arterial distensibility profile of subjects affected by Cushing's syndrome. Increased arterial stiffness may persist even after successful cure of this disease. It is therefore of fundamental importance to identify the presence of early vascular alterations in these patients, in order to commence their treatment and thus attempt to prevent the subsequent onset of adverse cardiovascular events.
\end{abstract}

\section{Keywords}

Arterial stiffness, cardiovascular risk, cardiovascular protection, Cushing's syndrome, hypertension, obesity, dyslipidaemia, the metabolic syndrome, endothelial function, ultrasound, plethysmography

Disclosure: Pier Paolo Bassareo, Angela Maria Zedda and Giuseppe Mercuro have no conflicts of interest to declare. No funding was received for the publication of this article. Received: 31 May 2013 Accepted: 7 August 2014 Citation: European Endocrinology, 2014;10(2):161-4 DOI:10.17925/EE.2014.10.02.161

Correspondence: Pier Paolo Bassareo, Department of Medical Sciences 'M Aresu', University of Cagliari, Policlinico Universitario, S.S. 554, bivio di Sestu - 09042 Monserrato, Cagliari, Italy. E: piercard@inwind.it

\section{Arterial Stiffness and Cardiovascular Risk}

Recently, much emphasis has been placed on the role of arterial stiffness in the development of cardiovascular diseases. In fact, increased arterial wall rigidity has been acknowledged as a major independent risk factor for cardiovascular pathologies, ${ }^{1,2}$ and is frequently correlated to the onset of adverse cardiovascular events., ${ }^{3,4}$ Representing a physiological hallmark of the ageing process, reduced arterial wall distensibility has been also observed in the presence of numerous pathological conditions, including hypertension, ${ }^{5}$ diabetes ${ }^{6}$ obesity, ${ }^{7}$ dyslipidaemia ${ }^{8}$ and kidney diseases. ${ }^{9}, 10$ Furthermore, it has been reported how impaired peripheral arterial distensibility may reflect coronary artery endothelial dysfunction as well. ${ }^{11}$

Indeed, aortic stiffening accompanying age and cardiovascular risk factors is caused by various phenomena, including breaks in elastin fibres, accumulation of collagen, fibrosis, inflammation, medial smooth muscle necrosis, calcifications and diffusion of macromolecules within the arterial wall. All these phenomena are known to occur in parallel at the site of the coronary circulation.

In the light of evidence provided, and of the suitability of non-invasive detection methods, such as photoplethysmography and ultrasonography, this marker of early arterial atherosclerosis is currently increasingly applied in the clinical assessment of patients. ${ }^{12}$

\section{Search Strategy}

A PubMed/Medline search was conducted using the MeSH terms: Cushing's syndrome, arterial stiffness, arterial compliance, arterial distensibility, intima-media thickness, hypophyseal adenoma, hypophysis, adrenocorticotrope hormone(s), glucocorticoid (GC) hormone(s), cortisol, ultrasound, echography, plethysmography, flow-mediated dilation (FMD), Doppler, cardiac catheterisation, cardiac computed tomography, cardiovascular risk, cardiovascular protection, hypertension, obesity, dyslipidaemia, the metabolic syndrome, endothelial function and their combinations. Articles identified in this manner were retrieved and reference lists searched for additional relevant articles. The search was limited to English-language publications, but no other restrictions were applied. The PubMed/Medline database was searched from its inception to May 2013. The most important of these papers are reported in this review.

\section{Cushing's Syndrome and Increased Cardiovascular Risk}

Cushing's syndrome is an endocrine disorder more frequent in females (female/male ratio = 8:1). It is characterised by the hypersecretion of cortisol, generally due to the presence of an adenocorticotrope hormone-secreting hypophyseal adenoma. Less common causes are primary adrenal diseases, ectopic adrenocorticotrope hormone secretions and exogenous assumption of corticosteroids

Cushing's syndrome is underpinned by a complex metabolic syndrome, which is potentially implicated in the onset of blood vessel alterations and an increase in arterial wall stiffness. The clinical picture is characterised by abdominal obesity, systemic arterial hypertension, impaired glucose metabolism, hyperlipidaemia and hypercoagulation of the blood. Taken together, these factors constitute an ideal substrate for the development of cardiovascular diseases, such as coronary heart disease, stroke and heart failure. Indeed, cardiovascular death in patients with active Cushing's 


\section{Pituitary Disorders}

syndrome is approximately three- or fourfold higher compared with the general population matched for age and gender. Furthermore, untreated Cushing's syndrome has a poor prognosis, with only a 50 \% 5-year survival. ${ }^{13-15}$

Numerous research projects have demonstrated how the excessive secretion of GC hormones, in particular cortisol, produces a direct effect on the cardiovascular system, leading to both activation of the renin-angiotensin system resulting in sympathetic hypertension, and a reduced release of nitric oxide by the endothelium. ${ }^{16}$ Although adrenocorticotrope hormones receptors have been demonstrated in human aortic endothelial cells, ${ }^{17}$ it seems unlikely their direct actions in the development of hypertension in Cushing's syndrome's subjects. ${ }^{15}$

Again, Philips et al. ${ }^{18}$ have reviewed evidence for the foetal programming of the neuroendocrine response to stress, thus highlighting the correlation of small size at birth with increased adrenocortical and sympatocoadrenal responses.

Since peroxisome proliferator-activated receptor agonists lower blood pressure in human subjects, ${ }^{19}$ it is interesting to observe their effects on downregulation of 11 beta-hydroxysteroid dehydrogenase, and hence availability of biologically active GCs. ${ }^{20}$

The complex metabolic alterations elicited by the onset of hypercortisolism strongly resemble those manifested by the metabolic syndrome. Indeed, similar to the metabolic syndrome, an acceleration of the atherosclerotic process, which may be manifested in the initial stages of the disease with increased arterial wall stiffness, is also observed in Cushing's syndrome. ${ }^{21}$ An excess of cortisol secretion has been indicated as a likely, and potentially curable, cause underlying the increased cardiovascular risk detected in patients with Cushing's syndrome. ${ }^{22}$

Central adiposity, which is one of the main characteristics of the metabolic syndrome, may reflect 'Cushing's disease of the omentum'. Adipose stromal cells from omental, but not from subcutaneous fat, can generate active cortisol from inactive cortisone through expression of 11 beta-hydroxysteroid dehydrogenase. In addition, this enzyme expression is increased after exposure to cortisol and insulin. Such a mechanism would ensure constant GC exposure specifically to omental fat, thus leading to central obesity. ${ }^{23}$

Glucose intolerance and diabetes are common features in Cushing's syndrome. In those subjects with hyperglycaemia, cortisol secretion although normal - appears to be inappropriately high, given enhanced peripheral sensitivity to GCs. ${ }^{24}$

Specific variations in GC receptor expression and function might explain information about the pathophysiology of insulin resistance in these individuals. ${ }^{25}$

Hypercholesterolaemia and hypertriglyceridaemia are common in Cushing's syndrome. ${ }^{26}$ In this respect, it has been reported that cortisol excretion is correlated negatively with high-density lipoprotein (HDL) cholesterol at multivariate analysis. ${ }^{27}$ Higher plasma cortisol is associated with higher tryglicerides in female gender, but not in males. ${ }^{28}$ Morning salivary cortisol is strongly associated with tryglicerides concentration as well. 29,30

In opposition to the above-stated reported reversibility of the cardiovascular system involvement in Cushing's syndrome, other studies have reported how vascular modifications induced by prolonged exposure to excessive secretion of glucocorticoids may be irreversible. These studies demonstrated that in patients with Cushing's disease arterial hypertension induced by endothelial alterations, may persist even after surgical treatment. This would seem to imply that in Cushing's disease the resolution of hypercortisolism despite the improvement observed in biochemical parameters - does not necessarily result in a decrease of cardiovascular risk. ${ }^{31}$

\section{Pathophysiology of Reduced Arterial Compliance}

Early atherosclerosis may contribute to the increased rates of cardiovascular morbidity and mortality in patients with GC excess. However, the mechanisms by which the excess of GC hormones induce vascular alterations and resulting arterial stiffness remain to be fully elucidated.

It has been demonstrated that glucocorticoids elicit a decrease at endothelial level, in nitric oxide synthase and enzymatic activity implicated in the release of nitric oxide, a major vasodilatory compound. ${ }^{32}$ Moreover, glucocorticoids inhibit transmembrane transport of arginine, the substrate for nitric oxide synthesis, and decrease the cofactor tetrahydriobiopterin.33,34 Additionally, glucocorticoids lead to an increase in oxidative stress through the production of hydrogen peroxide and peroxynitrite. ${ }^{35}$

Similarly, several other molecules would seem to be implicated in the manifestation of altered endothelial function in patients with Cushing's disease: adhesion molecules, homocysteine, osteoprotegerin, endothelin, cytokines and markers of inflammation. ${ }^{36-41}$

For example, it has been demonstrated that hyperhomocysteinaemia and reduced serum folate concentrations are associated with active hypercortisolism, whereas patients in Cushing's syndrome remission may have homocysteine concentrations comparable with healthy subjects. ${ }^{42}$ Low serum folate concentrations do not fully account for the increase in homocysteine levels that may play a pivotal role in the prothrombotic state and increased cardiovascular risk of these patients. ${ }^{42}$

Elevated plasma endothelin-1 and osteoprotegerin levels also appear to be involved in the increased coronary risk in these patients, being involved in the pathogenesis of an early and accelerated atherosclerosis development in these vessels. ${ }^{43,44}$

Glucocorticoid involvement in the regulation of vascular tone is accomplished by enhancing vasoconstrictor response to catecholamines and decreasing vasodilation and prostacyclin synthesis..$^{45}$ Cortisol likewise results in a blockade of vasodilatation routinely induced by the intraarterial infusion of acetylcholine. ${ }^{46}$

The above-listed represent only a few of the vastly complex mechanisms implicated in the pathogenesis of vascular remodeling in Cushing's syndrome. Long-term elevations in angiotensinogen concentration, which occur in Cushing's syndrome, may be a risk factor for hypertension, although there is evidence that chronic stimulation of angiotensinogen may be partly compensated for by a reduction in renin secretion. ${ }^{47}$

Among the numerous pathogenetic mechanisms proposed, one impinges on the aldosterone receptor, previously implicated in the pathogenesis of arterial hypertension with secondary hyperaldosteronism. Several animal models have indeed displayed an aldosterone-independent and GC receptor-dependent response mediated by aldosterone receptor signalling, resulting in the growth of smooth vascular muscle cells and 
consequent muscular hypertonia and hypertrophy of the tunica media. ${ }^{48,49}$ Vascular remodelling affects both large blood vessels and smaller arterioles. Rizzoni et al. highlighted how patients with Cushing's syndrome displayed structural alterations to the small arterioles, and, in particular, a decreased intima-media ratio, with hypertrophic remodelling resembling that observed in patients affected by essential hypertension..$^{50}$

Lastly, a relevant role would likewise appear to be played by sphingolipids. An accumulation in the endothelium of aberrant ceramides and other types of sphingolipids induced by cortisol, free fatty acids (FFA) and tumour necrosis factor-alpha (TNF- $\alpha$ ), determines a marked increase in arterial wall stiffness in these patients. ${ }^{5}$

\section{Arterial Stiffness Measured Using Ultrasonography}

In an important study conducted by Faggiano et al., carotid intima-media thickness, and other ecographic parameters of vascular distensibility (systolic and diastolic media-media distance, systolic lumen diameter diastolic lumen diameter, blood systolic and diastolic peak velocities and distensibility coefficient) were evaluated in patients affected by Cushing's syndrome (during the active phase and 1 year after treatment) and in a group of healthy controls. The study reported how patients with Cushing's syndrome presented with increased vascular stiffness (indicated by higher intima-media thickness associated with a decreased diastolic lumen diameter and a lower distensibility index) and increased presence of atherosclerotic plaques compared with controls. These alterations were still present 1 year following remission of the disease. The authors maintained that this occurrence was due to the persistence of factors, such as abdominal obesity and altered glucose metabolism. ${ }^{52}$

When the experiment was repeated 5 years after completion of treatment, the continuing presence of increased arterial stiffness was reiterated. Once again, underlying causes were represented by the persistence of abdominal obesity and onset of insulin resistance. ${ }^{53}$

Hypertrophic remodelling has been described also in subcutaneous small resistance arteries of patients affected by Cushing's syndrome, probably as a consequence of growth-promoting properties of circulating cortisol and/or increased vascular oxidative stress. ${ }^{54}$

\section{Arterial Stiffness Measured Using Plethysmography}

The so-called FMD method, assessing the ability of digital vascular endothelium to dilate in response to an ischaemic stimulus, is another of the several techniques used in the study of arterial stiffness. The latter ability is reduced in the presence of an endothelial dysfunction, representing the first sign of onset of an atherosclerotic process. A series of methods is available for use in determining FMD, among which plethysmography. ${ }^{55}$

In spite of the finding of a lack of statistically significant differences in intima-media thickness compared with controls, use of the above technique in Cushing's disease reveals a reduction in FMD ( $p=0.0014)$ However, this early condition - which conversely to an increased intimamedia thickness is statistically correlated to hypercortisolism but not to obesity and insulin resistance - can be reversed by means of medical and/or surgical correction of excess cortical secretion. Accordingly, in these patients this would seem to reflect the presence of a exclusively functional impairment rather than a dysfunction linked to the onset of anatomical modifications, such as atherosclerotic plaques.56
Similar results have been reported in studies undertaken to investigate brachial artery FMD by means of ultrasonography $(p<0.0001$, although no significant difference was revealed between the two groups with regard to baseline diameter of brachial artery). ${ }^{57}$

Assessment of endothelial function may thus identify high-risk individuals early, allow therapy to reduce or retard endothelial dysfunction to be started, and may lead to decreased cardiovascular morbidity and mortality in patients with suffering from Cushing's syndrome. ${ }^{58}$

\section{Arterial Stiffness Measured Using Other Methods} Endothelial function in patients with Cushing's syndrome can be measured using laser Doppler flowmetry method (skin microvascular reactivity) as well. This technique uses laser beam and fibre optics probes for detection of blood flow in small vessels located in adjacent layers of the tissue. Combination of laser Doppler flowmetry with an assay of biochemical markers of endothelial dysfunction, oxidative stress and fibrinolysis can bring up additional information on vascular changes. Based on these premises, it has been demonstrated that oxidative stress and endothelial dysfunction were present in patients affected by Cushing's syndrome.

Furthermore, increased concentration of ICAM-1 (a laboratory marker of endothelial dysfunction) was also found in patients after the operation compared with controls, thus underlying an only partial reversible endothelial damage. The most pronounced impairment of skin microvascular reactivity was present in Cushing's patients with combination of arterial hypertension and diabetes mellitus. ${ }^{59}$

By using coronary angiography, it has been recently demonstrated that increased coronary calcifications and non-calcified coronary plaque volumes are present in patients with active hypercortisolism. However, this preliminary case-control study was based on a small sample (only 15 consecutive patients). ${ }^{60}$

The prevalence of coronary artery disease has been also evaluated in Cushing's syndrome patients after long-term remission using multidetector computer tomography coronary angiogram scan, which demonstrated that patients in remission of hypercortisolism (for a mean duration of 11 years) are still at high risk of cardiovascular disease, especially women and younger patients. ${ }^{61}$

This was a further evidence that increased cardiovascular risk persists in Cushing's syndrome despite remission of hypercortisolism.

\section{Arterial Stiffness in Paediatric Patients Suffering from Cushing's Syndrome}

Our research group has previously investigated arterial stiffness in young female patients (age range 11-18 years) with Cushing's disease. Two studies conducted in this context demonstrated comparable results to those obtained in adults. In particular, in a study of young females we demonstrated the presence of an increased intima-media thickness compared with healthy controls, both prior to and following surgical treatment. ${ }^{2}$ It should be underlined how an increased intima-media thickness in young people is a widely acknowledged risk factor for the future development of atherosclerosis in adulthood. ${ }^{63}$

In a further study conducted on the same young patients, we carried out 24-hour monitoring of vascular distensibility using the so-called quantum key distribution (QKd) method. 
QKd interval is the time (measured in millisecond) between the onset of depolarisation on the electrocardiogram (Q) and detection of the last Korotkoff sound $(K)$ at the level of the brachial artery during cuff deflation, which corresponds to diastolic blood pressure (d). As this interval is inversely related to pulse wave velocity, the QKd measurement provides valuable information about 24-hour arterial distensibility.

In fact, while the above-stated methods provide an evaluation only at the time of examination, the QKd interval technique provides a complete profile of circadian variations in pulse wave velocity that would be more appropriate than a single measurement in assessing arterial elasticity.

Thus, a statistically significant difference was obtained to the detriment of the young patients affected by Cushing's syndrome compared with controls $(p<0.01)$, in addition to the finding of higher blood pressure values during 24-hour monitoring. ${ }^{64}$

\section{Conclusions}

To conclude, it has been demonstrated that in Cushing's syndrome vascular alterations are based on complex pathogenetic mechanisms, undoubtedly correlated to the metabolic alterations directly induced by hypercortisolism, influencing in particular the production and release of endothelial nitric oxide. Persistence of these alterations produces a significant effect on cardiovascular mortality of these patients; medical and/or surgical treatment of the disease, even when successful, does not unfortunately result in a marked reduction of this risk. Duration of the disease likewise appears to constitute an important factor in determining a protractedly increased cardiovascular risk induced by Cushing's syndrome. It is therefore of fundamental importance to identify the presence in these patients of early vascular alterations, including arterial wall stiffness, in order to commence treatment of the alterations and thus attempt to prevent the subsequent onset of adverse cardiovascular events.
1. Benetos A, Safar M, Rudnichi A, et al., Pulse pressure: a predictor of long-term cardiovascular mortality in a French male population, Hypertension, 1997;30:1410-5.

2. Benetos A, Rudnichi A, Safar M, Guize L, Pulse pressure and cardiovascular mortality in normotensive and hypertensive subjects, Hypertension, 1998;32:560-4.

3. Mitchell GF, Moyé LA, Braunwald E, et al., Sphygmomanometrically determined pulse pressure is a powerful independent predictor of recurrent events after myocardial infarction in patients with impaired left ventricular function, Circulation, 1997;96:4254-60.

4. Chae CU, Pfeffer MA, Glynn RJ, et al., Increased pulse pressure and risk of heart failure in the elderly, JAMA, 1999;281:934-9.

5. Laurent $\mathrm{S}$, Boutouyrie $\mathrm{P}$, Asmar R, et al., Aortic stiffness is an independent predictor of all-cause and cardiovascular mortality in hypertensive patients, Hypertension, 2001;37:1236-41

6. Jaiswal M, Urbina EM, Wadwa RP, et al., Reduced heart rate variability is associated with increased arterial stiffness in youth with type 1 diabetes: The SEARCH Cardiovascular Disease Study, Diabetes Care, 2013;36:2351-8.

7. Steinberg $\mathrm{HO}$, Chaker $\mathrm{H}$, Leaming R, et al., Obesity/insulin resistance is associated with endothelial dysfunction. Implications for the syndrome of insulin resistance, $J$ Clin Invest 1996;97:2601-10

8. Akalin A, Temiz G, Akcar N, Sensoy B, Short term effects of atorvastatin on endothelial functions and oxidized LDL levels in patients with type 2 diabetes, Endocr J, 2008;55:861-6.

9. Blacher J, Guerin AP, Pannier B, et al., Impact of aortic stiffness on survival in end-stage renal disease, Circulation, stiffness on survival.

10. Blacher J, Pannier B, Guerin A, et al., Carotid arterial stiffness as a predictor of cardiovascular and all-cause mortality in end stage renal disease, Hypertension, 1998;32:570-4.

11. Anderson TJ, Uehata A, Gerhard MD, et al., Close relation of endothelial function in the human coronary and peripheral circulations, J Am Coll Cardiol, 1995;26:1235-41.

12. Laurent $S$, Cockcroft J, Van Bortel L, et al., Expert consensus document on arterial stiffness: methodological issues and clinical applications, Eur Heart J, 2006;27:2588-605.

13. Aron DC, Findling JW, Tyrrell JB, Cushing's disease, Endocrinol Metab Clin North Am, 1987;16:705-30.

14. Etxabe J, Vazquez JA, Morbidity and mortality in Cushing's disease: an epidemiological approach, Clin Endocrinol (Oxf), 1994:40:479-84.

15. Whitworth JA, Williamson PM, Mangos G, Kelly JK, Cardiovascular consequences of cortisol excess, Vasc Health Risk Manag, 2005;1:291-99.

16. Saruta T, Mechanism of glucocorticoid-induced hypertension, Hypertens Res, 1996;19:1-8

17. Hatakeyama H, Inaba S, Taniguchi N, Miyamori I, Functional adrenocorticotropic hormone receptor in cultured human vascular vascular endothelial cells: possible role in control of blood pressure, Hypertension, 2000;36:862-5.

18. Philips DI, Fetal programming of the neuroendocrine reonse to stress: link between low birth weight and the metabolic syndrome, Endocr Res, 2004;0:819-26.

19. Hirose H, Kawai T, Yamamoto Y, et al., Effects of pioglitazone on metabolic parameters, body fat distribution, and serum adiponectin levels in Japanese male patients with type 2 diabetes, Metabolism, 2002;51:314-17.

20. Berger J, Tanen M, Elbrecht A, et al., Peroxisome proliferatoractivated receptor-gamma ligands inhibit adipocyte 11beta -hydroxysteroid dehydrogenase type 1 expression and activity J Biol Chem, 2001;276:12629-35.

21. Chanson P, Salenave S, Metabolic syndrome in Cushing's syndrome, Neuroendocrinology, 2010;92 Suppl. 1:96-10

22. Walker BR, Cortisol - cause and cure for metabolic syndrome?, Diabet Med, 2006;23:1281-8.

23. Bujalska IJ, Kumar S, Stewart PM, Does central obesity reflect Cushing's disease of the omentum?, Lancet, 1997;349:1210-3.
24. Andrews RC, Herlihy O, Livingstone DE, et al., Abnormal cortisol metabolism and tissue sensitivity to cortisol in patients with glucose intolerance, I Clin Endocrinol Metab, 2002;87:5587-93

25. Reynolds RM, Chapman KE, Seckl JR, et al., Skeletal muscle glucocorticoid receptor density and insulin resistance, JAMA 2002;287:2505-6

26. Arnaldi G, Mancini T, Tirabassi G, et al., Advances in the epidemiology, pathogenesis, and management of Cushing's syndrome complications, J Endocrin Invest, 2012;35:434-38.

27. Fraser $\mathrm{R}$, Ingram $\mathrm{MC}$, Anderson $\mathrm{NH}$, et al., Cortisol effects on body mass, blood pressure, and cholesterol in the general population, Hypertension, 1999;33:1364-8.

28. Walker BR, Soderberg S, Lindahl B, Olsson T, Independent effects of obesity and cortisol in predicting cardiovascular risk effects of obesity and cortisol in predicting cardiovascular risk

29. Wallerius S, Rosmond R, Ljung T, et al., Rise in morning saliva cortisol is associated with abdominal obesity in men: a preliminary report, Endocrinol Invest, 2003;26:616-9.

30. Ward AM, Fall CH, Stein CE, et al., Cortisol and the metabolic syndrome in South Asians, Clin Endocrinol (Oxf), 2004;58:500-5.

31. Sacerdote A, Weiss $K$, Tran T, et al., Hypertension in patients with Cushing's disease: pathophysiology, diagnosis, and management, Curr Hypertens Rep, 2005;7:212-8.

32. Wallerath T, Witte K, Schafer SC, et al., Down-regulation of the expression of endothelial NO synthase is likely to contribute to glucocorticoid-mediated hypertension, Proc Natl Acad Sci U S A, 1999; 96:13357-62.

33. Simmons WW, Ungureanu-Longrois $D$, Smith GK, et al., Glucocorticoids regulate inducible nitric oxide synthase by inhibiting tetrahydrobiopterin synthesis and L-arginine by inhibiting tetrahydrobiopterin synthesis
transport, J Biol Chem, 1996;271:23928-37.

34. Mitchell BM, Dorrance AM, Mack EA, Webb RC, Glucocorticoids decrease GTP cyclohydrolase and tetrahydrobiopterindependent vasorelaxation through glucocorticoid receptors, J Cardiovasc Pharmacol, 2004;43:8-13.

35. Iuchi T, Akaike M, Mitsui T, et al., Glucocorticoid excess induces superoxide production in vascular endothelial cells and elicits vascular endothelial dysfunction, Circ Res, 2003;92:81-7.

36. Terzolo M, Allasino B, Bosio S, et al., Hyperhomocystinemia in patients with Cushing's syndrome, I Clin Endocrinol Metab 2004;89:3745-51.

37. Ermetici F, Malavazos AE, Corbetta S, et al., Soluble adhesion molecule levels in patients with Cushing's syndrome before and after cure, J Endocrinol Invest, 2008:31:389-92.

38. Kristo $\mathrm{C}$, Ueland $\mathrm{T}$, Godang $\mathrm{K}$, et al., Biochemical markers for cardiovascular risk following treatment in endogenous Cushing's syndrome, J Endocrinol Invest, 2008:31:400-5.

39. Camozzi V, Sanguin F, Albigier N, et al., Persistent increase of osteoprotegerin levels after cortisol normalization in patients with Cushing's syndrome, Eur J Endocrinol, 2010;162:85-90

40. Kirilov G, Tomova A, Dakovska L, et al., Elevated plasma endothelin as an additional cardiovascular risk factor in patients with Cushing's syndrome, Eur J Endocrino 2003;149:549-53.

41. Zacharieva S, Atanassova I, Nachev E, et al., Markers of vascular function in hypertension due to Cushing's syndrome Horm Metab Res, 2005;37:36-9.

42. Terzolo M, Allasino B, Bosio S, et al., Hyperhomocysteinemia in patients with Cushing's syndrome, J Clin Endocrinol Metab, 2004:89:3745-51.

43. Kirilov G, Tomova A, Dakovska L, et al., Elevated plasma endothelin as an additional cardiovascular risk factor endothelin as an additional cardiovascular risk factor in patients with C

44. Dovio A, Allasino B, Palmas E, et al., Increased osteoprotegeri levels in Cushing's syndrome are associated with an adverse cardiovascular risk profile, I Clin Endocrinol Metab 2007;92:1803-8.

45. Yang S, Zhang L, Glucocorticoids and vascular reactivity, Curr Vasc Pharmacol, 2004;2:1-12.
46. Mangos GJ, Walker BR, Kelly JJ, et al., Cortisol inhibits colinergic vasodilation in the human forearm, Am I Hypertens, 2000;13:1155-60.

47. Atlas SA, The renin-angiotensin aldosterone system: pathophysiological role and pharmacologic inhibition, J Manag Care Pharm, 2007;13(8 Suppl. b):9-20.

48. van den Meiracker AH, Batenburg WW, Corticosteroiddependent, aldosterone-independent mineralocorticoidreceptor activation in the heart, J Hypertens, 2008;26:1307-9.

49. Wang H, Shimosawa T, Matsui H, et al., Paradoxical mineralocorticoid receptor activation and left ventricular diastolic dysfunction under high oxidative stress conditions, $J$ Hypertens, 2008;26:1453-62.

50. Rizzoni D, Porteri E, De Ciuceis C, et al.. Hypertrophic remodeling of subcutaneous small resistance arteries in
rement patients with Cushing's syndrome, J Clin Endocrinol Metab, patients with Cusir

51. Summers $\mathrm{SA}$, Nelson $\mathrm{DH}$, A role for sphingolipids in producing the common features of type 2 diabetes, metabolic syndrome $X$, and Cushing's syndrome, Diabetes, 2005;54:591-602.

52. Faggiano A, Pivonello R, Spiezia S, et al., Cardiovascular risk factors and common carotid artery caliber and stiffness in patients with Cushing's disease during active disease and 1 year after disease remission, J Clin Endocrinol Metab 2003;88:2527-33.

53. Colao A, Pivonello R, Spiezia S, et al., Persistence of increased cardiovascular risk in patients with Cushing's disease after five years of successful cure, I Clin Endocrinol Metab, 1999;84:2664-72.

54. Rizzoni D, Porteri E, De Ciuceis C, et al., Hypertrophic remodeling of subcutaneous small resistance arteries in patients with Cushing's syndrome, J Clin Endocrinol Metab, 2009;94:5010-8.

55. Bassareo PP, Fanos V, Puddu M, et al., Reduced brachia flow-mediated vasodilation in young adult ex extremely low birth weight preterm: a condition predictive of increased cardiovascular risk?, J Matern Fetal Neonatal Med, 2010;23(Suppl. 3):121-4.

56. Akaza I, Yoshimoto T, Tsuchiya K, Hirata Y, Endothelial dysfunction associated with hypercortisolism is reversible in Cushing's syndrome, Endocrine J, 2010;57:245-52.

57. Baykan M, Erem C, Gedikli O, et al., Impairment of flowmediated vasodilatation of brachial artery in patients with Cushing's Syndrome, Endocrine, 2007:31:300-4.

58. Baykan M, Erem C, Gedikli O, et al., Impairment of flowmediated vasodilatation of brachial artery in patients with Cushing's Syndrome, Endocrine, 2007:31:300-4.

59. Prázný M, Jezková J, Horová E, et al., Impaired microvascular reactivity and endothelial function in patients with Cushing's syndrome: influence of arterial hypertension, Physiol Res, 2008;57:13-22

60. Neary NM, Booker OJ, Abel BS, et al., Hypercortisolism is associated with increased coronary arterial atherosclerosis analysis of noninvasive coronary angiography using multidetector computerized tomography, I Clin Endocrino Metab, 2013;98:2045-52.

61. Barahona MJ, Resmini E, Viladés D, et al., Coronary artery disease detected by multislice computed tomography in patients after long-term cure of Cushing's syndrome, I Clin Endocrinol Metab, 2013:98:1093-9.

62. Bassareo PP, Fanos V, Zaffanello M, Mercuro G, Early markers of cardiovascular dysfunction in young girls affected by Cushing's syndrome before and after successful cure, Cushing's syndrome before and after success
Pediatr Endocrinol Metab, 2010:23:627-35.

63. Freedman DS, Dietz WH, Tang R, et al., The relation of obesity throughout life to carotid intima-media thickness in adulthood: the Bogalusa Heart Study, Int I Obes Relat Metab Disord, 2004;28:159-66

64. Bassareo PP, Marras AR, Pasqualucci D, Mercuro G, Increased arterial rigidity in children affected by Cushing's syndrome after successful surgical cure, Cardiol Young, 2010;20:610-4. 